OPEN ACCESS

Edited by:

Slobodan Paessler

University of Texas Medical Branch,

USA

Reviewed by:

César López-Camarillo,

Universidad Autonoma de la Ciudad

de Mexico, Mexico

Irma Elisa Cisneros,

University of Texas Medical Branch

USA

*Correspondence:

Shu Yuan

roundtree318@hotmail.com

Received: 02 August 2016 Accepted: 22 December 2016 Published: 09 January 2017

Citation:

Zhang Z-W, Li Z-L and Yuan S (2017)

The Role of Secretory Autophagy in Zika Virus Transfer through the Placental Barrier.

Front. Cell. Infect. Microbiol. 6:206. doi: 10.3389/fcimb.2016.00206

\section{The Role of Secretory Autophagy in Zika Virus Transfer through the Placental Barrier}

\author{
Zhong-Wei Zhang ${ }^{1}$, Zi-Lin $\mathrm{Li}^{2}$ and Shu Yuan ${ }^{1 *}$ \\ ${ }^{1}$ College of Resources, Sichuan Agricultural University, Chengdu, China, ${ }^{2}$ General Hospital of Lanzhou Military Region, \\ Lanzhou, China
}

Recent studies indicated that the Zika virus genome could be detected in the amniotic fluid and the fetal brain, which confirms that the virus can cross the placental barrier. Secretory autophagy or exosome pathways may participate in this virus transfer. Autophagy modulators regulate autophagosome formation or membrane fusion with Iysosomal vesicles and therefore inhibit viral nucleocapsid releasing or virus transfer to the fetus hypothetically. However, some autophagy modulators may enhance virus replication. Autophagy inhibitors may arrest placental development; while exaggeration of autophagy in human placenta may be associated with the fetal growth restriction. Therefore, autophagy modulators should be used carefully due to their complex clinical effects. Alternatively, exosome-specific inhibitors might be also considered, although their safety of both maternal and fetal conditions must be carefully assessed before any advancement to human clinical trials.

Keywords: Zika virus, microcephaly, secretory autophagy, exosome, placental barrier

\section{INTRODUCTION}

A widespread epidemic of Zika virus (an emerging mosquito-borne flavivirus) infections was found in Central and South America recently. The main concern is the significant increasing of microcephaly in the fetus born to the mother of Zika virus infection (Faria et al., 2016). Placenta may play an important role in the virus transfer. One possibility is that the Zika virus can penetrate through the placental barrier. Alternatively, the infection to the placenta might induce some immune responses and cause brain defects indirectly (Adibi et al., 2016a). A recent study indicated that the viral genome could be detected in the amniotic fluid, which confirms that the virus could penetration through the placental barrier (Calvet et al., 2016). The complete genome of Zika virus can be also recovered from the fetal brain, which confirms the tropism of Zika virus for neural tissues (Mlakar et al., 2016). Direct evidences of fluorescence in situ hybridization (FISH) confirmed the presence of Zika virus RNA in mutiple mouse trophoblast cells, such as glycogen trophoblasts, mononuclear trophoblasts, spongio-trophoblasts, and syncytiotrophoblasts. Transmission electron microscopy of the mouse placenta found many 50-nm bodies in the endoplasmic reticulum of trophoblast cells (Miner et al., 2016). Indirect immunoblotting with the serum obtained from the infected mother found fluorescence signals in the destroyed neuron cells. And negative staining of the infected fetal brain tissue showed $42-54 \mathrm{~nm}$ spherical virus particles (Mlakar et al., 2016). Therefore the hypothesis that the virus could penetration through the placental barrier has been confirmed. 


\section{SECRETORY AUTOPHAGY MAY MEDIATE ZIKA VIRUS TRANSFER}

However, we still do not know by what exact mechanism Zika virus transfers through the trophoblast cell. Like dengue virus, Zika virus may be packaged as a cargo for the placental exosome pathway at the trophoblast endoplasmic reticulum, which is closely associated with the "secretory autophagy" process (Chahar et al., 2015; Carneiro and Travassos, 2016). Autophagy usually has a protective role by removing damaged organelles and protein aggregates and maintaining cellular homeostasis (Ponpuak et al., 2015). Contrasting to the degradative autophagy, there is also a non-degradative autophagic machinery, termed secretory autophagy (Ponpuak et al., 2015). Some proteins lack the $\mathrm{N}$-terminal leader peptides, and therefore cannot enter the common endoplasmic-reticulum-to-Golgi-apparatus secretory pathway. And these proteins (such as viral capsid polypeptides) may be exported through the secretory autophagy pathway unconventionally (Ponpuak et al., 2015). Different from the degradative autophagy (fusion with the lysosome), the secretory autophagy pathway may cause expulsion or secretion of the viral particle other than its degradation (Figure 1). Zika virus infection is associated with the observation of a lot of double membrane intracytoplasmic vacuoles (namely the autophagosome). While the co-detection of the virus envelope peptides and the autophagy marker protein LC3 (cytosolic microtubule-associated light chain 3) has been reported (Hamel et al., 2015). Therefore, secretory autophagy (or exosome) may facilitate Zika virus transfer across the placental barrier, and regulations to the equilibrium between degradative autophagy and secretory autophagy may influence the incidence of microcephaly.

\section{AUTOPHAGY INHIBITORS AND THE CLINICAL EFFECTS}

For some flaviviruses, upon binding to the receptor, the virus enters the early endosome via the clathrin-mediated endocytic process (Nour et al., 2013; Carneiro and Travassos, 2016). About 5 min later, the virus particle fuses with the endosomal carrier vesicle (ECV) membrane predominantly (Figure 1). However for several minutes, viral nucleocapsids remain trapped in the ECV lumen, until ECVs fuse back with the late endosome limiting-membrane. These fusion events require microtubule movement, some membrane fusion factors and the PI(3)Pmediated signaling (Nour et al., 2013). PI(3)P kinase inhibitors, 3-methyladenine and wortmannin, inhibit both autophagosome formation, and ECV formation (Nour et al., 2013). In the absence of ECVs, the virus either fails to fuse fully with the early endosome (only partial fusion to the proximal membrane) or it fuses with some other endosomal vesicles but the nucleocapsid still is trapped (Figure 1; Nour et al., 2013). Autophagosomes were observed in human skin fibroblast cells infected with Zika virus (Hamel et al., 2015). The specific autophagy inhibitor 3-methyladenine strongly reduces Zika viral copy numbers in the infected fibroblast cells (Figure 1 and Table 1; Hamel et al., 2015). However, neither 3-methyladenine nor wortmannin is an FDA (the Food and Drug Administration) -approved drug.

The cellular alkalizers also repress degradative autophagy through neutralizing lysosomal acidic $\mathrm{pH}$, which is necessary for lysosomal hydrolases activations (Vakifahmetoglu-Norberg et al., 2015). Chloroquine and its derivative hydroxychloroquine are such alkalizers and are used clinically as anticancer drugs or antimalarial candidate medicines (Vakifahmetoglu-Norberg et al., 2015). Chloroquine had inhibitory effects on flavivirus replication in vitro and significantly decreased Dengue virus type 2 replication in Aotus monkeys (Farias et al., 2015), but it did not reduce the duration of viral infection in a human clinical trial and showed several adverse effects, primarily vomiting (Tricou et al., 2010). More importantly, chloroquine seems not to be related with the equilibrium between degradative autophagy and secretory autophagy (Figure $\mathbf{1}$ and Table $\mathbf{1}$ ).

In general, viruses customize autophagy protein for efficient viral entry (Pirooz et al., 2014). However, some opposite reports suggest that the involvement of autophagy in flavivirus infection is controversial. ATG16L2 (Autophagy related 16like 2) was identified among the top 30 genes down-regulated in human neural stem cells infected with Zika virus (Rolfe et al., 2016). LC3 transcript was also down-regulated by Zika virus infection in mice brain cells ( $\mathrm{Li}$ et al., 2016). However, West Nile virus infection did not induce LC3 lipidation in multiple mammalian cell lines (Vandergaast and Fredericksen, 2012). And depletion of autophagy-related (ATG) protein ATG5 does not affect replication of West Nile virus (Vandergaast and Fredericksen, 2012; Martín-Acebes et al., 2015). For mouse embryonic fibroblast cells infected with Japanese encephalitis virus, either depletion in ATG7 or deficiency in ATG5 would result in higher viral replication levels (Sharma et al., 2014). Autophagy may play a positive role in the early infection stages, however it becomes dysfunctional when the misfolded proteins accumulate at the late stages. Autophagy-deficient cells were highly susceptible to virus-induced cell death (Sharma et al., 2014). Therefore, the role of autophagy may be varied at different infection stages. Autophagy inhibitors should be used carefully because of their complex clinical effects.

The autophagy markers LC3-II and Beclin-1 are highly expressed in villous cytotrophoblasts at the first trimester and they are prevalently activated in trophoblast cells during the whole gestation period (Chifenti et al., 2013). The cytotrophoblast and the syncytiotrophoblast, which form the placental barrier, function differently according to different gestational ages. Autophagy plays an important role in the placental development and pregnancy maintaining (Gong and Kim, 2014). Therefore, autophagy inhibitors may produce adverse effects to the fetus.

\section{AUTOPHAGY STIMULATORS AND THE CLINICAL EFFECTS}

Metformin, a type 2 diabetes drug, induces autophagy by activating AMPK (adenosine monophosphate-activated kinase) pathways, may enhance antigen presentation and processing 


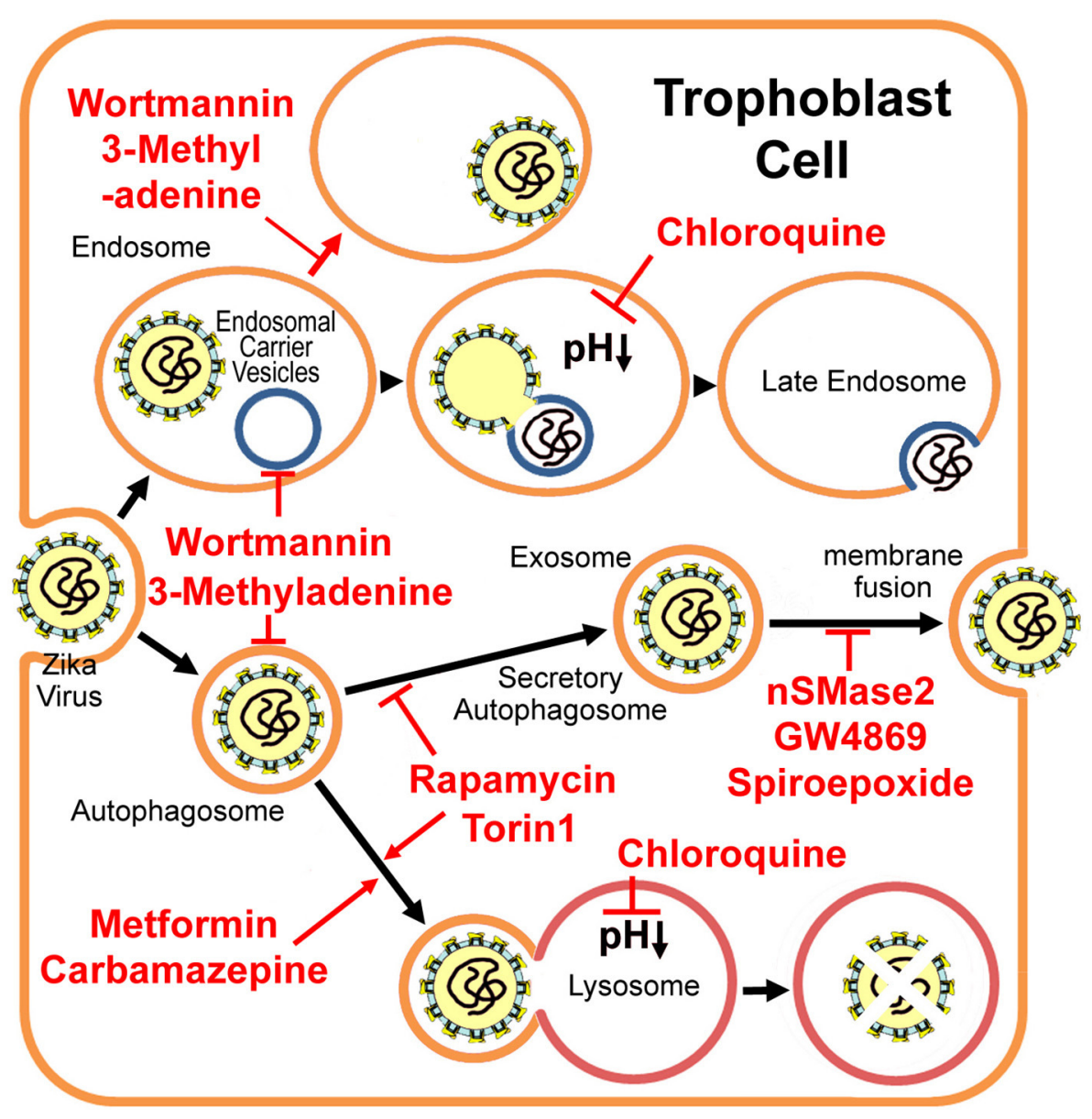

FIGURE 1 | Putative Zika virus entry pathway and the secretory autophagy/exosome pathway in the trophoblast cell. 3-methyladenine and wortmannin inhibit both autophagosome formation and endosomal carrier vesicle formation (Nour et al., 2013). In the absence of these vesicles, Zika virus may either fail to fuse completely with the early endosome membrane or it may fuse with as yet unidentified endosomal compartments in which the nucleocapsid remains trapped (Nour et al., 2013). Chloroquine and its derivative hydroxychloroquine are lysosomal lumen alkalizers, which inhibit degradative autophagy by neutralizing the acidic pH in the lumen of lysosomal vesicles (Vakifahmetoglu-Norberg et al., 2015). Metformin (Fedson, 2013) and Carbamazepine (Schiebler et al., 2015) induce autophagic killing of pathogenic microbes (degradative autophagy). mTORC inhibitors Torin 1 and rapamycin stimulate autophagy, but inhibit exosome release evidently (Fader et al., 2008), therefore limiting Zika virus transfer through the placental barrier. However, Torin 1 and rapamycin may enhance virus replication (Hamel et al., 2015). Alternatively, exosome-specific inhibitors nSMase2, GW4869 and spiroepoxide (Li et al., 2013) may prevent the transfer, although their drug safeties need further studies.

and improve maintenance of memory CD8 cytotoxic T lymphocytes after influenza virus infections (Fedson, 2013). Carbamazepine, an anticonvulsant drug, was indicated to trigger inositol-depletion dependent degradative autophagy of intracellular Mycobacterium tuberculosis (a pathogenic bacteria) in macrophages, which relieves pulmonary symptoms and activate mouse's immunity against the bacterium (Schiebler et al., 2015). Statin (a lipid-lowering medicine) reduced the M. tuberculosis proliferation in human and mouse macrophage cells through promoting phagosome maturation (Parihar et al., 2014). Membrane remodeling and viral replication usually induce the endoplasmic-reticulum stresses that cause the unfoldedprotein responses (Blázquez et al., 2014). Concomitant with the induction of the unfolded protein response, flavivirus infections induced autophagy-related pathway activation has been also described (Blázquez et al., 2014). For the instance of West Nile virus, stimulation of autophagy via apro-autophagic peptide
(Tat-beclin 1-derived from a region of the autophagy protein beclin 1) can protect neuronal cells from cell death caused by the virus infection (Shoji-Kawata et al., 2013). Thus, drugs that promote autophagy might have broad therapeutic applications (Table 1; Zumla et al., 2016).

Among the common autophagy stimulators, only rapamycin (a mTORC1 inhibitor) has been shown to inhibit exosome release evidently (Fader et al., 2008). Given that exosome may play a key role in Zika virus transfer, rapamycin might limit Zika virus transfer through the placental barrier. However, Torin 1 (another mTORC inhibitor) greatly enhances Zika virus replication (Hamel et al., 2015). Thus, the rapamycin may not be used as an efficient drug for the treatment of Zika virus infection.

Furthermore, misregulated autophagy may result in adverse consequences to the fetus. For example, exaggeration of autophagy in human placenta is associated with the fetal growth restriction (Curtis et al., 2013). Therefore, autophagy stimulators 
TABLE 1 | List of autophagy and exosome modulators and the clinical effects to virus or bacterial infections.

\begin{tabular}{|c|c|c|}
\hline Drug's name & Pathways involved in & Clinical effects \\
\hline $\begin{array}{l}\text { 3-Methyladenine } \\
\text { Wortmannin }\end{array}$ & PI(3)P kinase inhibitors; Autophagy inhibitors & $\begin{array}{l}\text { Reduce Zika viral copy numbers in the infected fibroblast cells } \\
\text { (Hamel et al., 2015) }\end{array}$ \\
\hline $\begin{array}{l}\text { Chloroquine } \\
\text { Hydroxychloroquine }\end{array}$ & Lysosomal lumen alkalizers; Autophagy inhibitors & $\begin{array}{l}\text { Decrease Dengue virus type } 2 \text { replication in Aotus monkeys (Farias } \\
\text { et al., 2015) }\end{array}$ \\
\hline Metformin & AMPK activator; Autophagy stimulator & $\begin{array}{l}\text { Enhances antigen processing and cytotoxic T lymphocytes after } \\
\text { influenza virus infections (Fedson, 2013) }\end{array}$ \\
\hline Carbamazepine & Induce inositol depletion -dependent autophagy & $\begin{array}{l}\text { Kill intracellular Mycobacterium tuberculosis; reduce tuberculosis } \\
\text { (Schiebler et al., 2015) }\end{array}$ \\
\hline Statin & Enhancing autophagy and phagosome maturation & $\begin{array}{l}\text { Reduces the Mycobacterium tuberculosis burden in human } \\
\text { macrophages (Parihar et al., 2014) }\end{array}$ \\
\hline Apro-autophagic peptide Tat-beclin 1 & Induce endoplasmic reticulum stress-associated autophagy & $\begin{array}{l}\text { Protect against neuronal cell death induced by the West Nile virus } \\
\text { infection (Shoji-Kawata et al., 2013) }\end{array}$ \\
\hline $\begin{array}{l}\text { Torin } 1 \\
\text { Rapamycin }\end{array}$ & mTORC1 inhibitor; Autophagy stimulator & $\begin{array}{l}\text { May prevent Zika virus transfer through the placental barrier (Fader } \\
\text { et al., 2008), but increase virus replication (Hamel et al., 2015) }\end{array}$ \\
\hline $\begin{array}{l}\text { nSMase2 } \\
\text { GW4869 } \\
\text { spiroepoxide }\end{array}$ & Neutral sphingomyelinase inhibitor; Exosome inhibitor & $\begin{array}{l}\text { May prevent Zika virus transfer through the placental barrier ( } \mathrm{Li} \\
\text { et al., 2013) }\end{array}$ \\
\hline
\end{tabular}

should also be chosen carefully due to their complex clinical effects.

\section{EXOSOME-SPECIFIC INHIBITORS}

Until now, the roles of autophagy in replication and secretion have not been well-established as contradictory results have been reported for different viruses. Hereby, some drugs other than autophagy modulators should also be considered, such as exosome-specific inhibitors nSMase2, GW4869, and spiroepoxide (neutral sphingomyelinase inhibitors; Table 1; Li et al., 2013). Sphingomyelinase and related ceramide play important roles in embryo development and apoptosis (de Castro e Paula and Hansen, 2008). However, alkaline sphingomyelinase and neutral sphingomyelinase are functionally similar. Neutral sphingomyelinase inhibitors might not cause significantly adverse consequences to the fetus, because of the complementation of alkaline sphingomyelinase. Nevertheless during pregnancy, when the number of available drugs is exceedingly limited and the bar for approval is extremely high, we must be very cautious when testing any potential therapeutic that could be used in human pregnancy. The safety of these exosome inhibitors must be carefully assessed (especially in non-human primates) before any advancement to human clinical trials. Alternatively, new exosome inhibitors should be developed, such as some natural compounds from soil actinomycetes (Dong et al., 2005). Then their safety at both maternal and fetal conditions requires further studies.

\section{POTENTIAL COMBINATIONS WITH OTHER DRUGS}

We should keep in mind that the secretory autophagy (exosome) may be only one of the complex mechanisms of Zika virus penetration through the placental barrier. Zika virus infection triggers apoptosis and vascular damages in the placenta, which may increase the permeability of the placenta (Miner et al., 2016). Therefore, Zika virus might be vertically transmitted independent of the secretory autophagy (exosome) pathway. A combination of antiviral agents and exosome inhibitors might be required to achieve the desired effect. For example, both type I interferons and type III interferons are induced by Zika virus infections (Bayer et al., 2016; Quicke et al., 2016), and interferon- $\lambda$ restricts Zika virus replication in human trophoblast cells (Bayer et al., 2016). Although, interferons and associated cytokines may generate indirect teratogenic and/or neurotoxic effects to the fetus (Adibi et al., 2016b; Mor, 2016), interferon- $\alpha$ does not apparently increase the risk of miscarriage, major malformation, preterm delivery, or stillbirth (Yazdani Brojeni et al., 2012). Moreover, interferon- $\alpha$ may show some protective effects against pregnancy loss in the case of essential thrombocythemia (Yazdani Brojeni et al., 2012).

When Zika virus has penetrated the placenta, it may damage the fetal brain directly or indirectly, because of its neurotropic property (Mlakar et al., 2016). This assumption implies that the virus exists in the fetal cerebral cortex at the early gestation stages. However, at that time, the fetus is fairly-well shielded from maternal circulation. Maternal blood flows into the placenta only after 10 weeks of gestation (Burton et al., 2001). Consistent with these structure changes, a tissue-level analysis in a case where the mother was infected at 7 weeks and miscarried at 12 weeks confirmed that the trophoblast was not infected by Zika virus at that period (Noronha et al., 2016). When Zika virus reaches trophoblast cells, non-neutralizing antibody and the virus may form an immuno-complex (Toth et al., 1994). And the exosome may be formed at that time, which may help the immuno-complex to transfer through the placental barrier. Nevertheless, the transfer is unlikely to be happened before 16 weeks of gestation (Saji et al., 1999; Fuchs and Ellinger, 2004). Therefore, autophagy-related drugs or exosome inhibitors may be not effective for the infected pregnant woman before 16 weeks. Instead, for these patients, interferons may help their immune system to clear the virus (Bayer et al., 2016; Quicke et al., 2016). 
Autophagy modulators or exosome inhibitors may be more useful to the medium-late stage pregnant women.

\section{AUTHOR CONTRIBUTIONS}

$\mathrm{ZZ}$ contributed to the discussion of ideas and helped with the writing. ZL contributed to the discussion of ideas and the

\section{REFERENCES}

Adibi, J. J., Marques, E. T. Jr., Cartus, A., and Beigi, R. H. (2016a). Teratogenic effects of the Zika virus and the role of the placenta. Lancet 387, 1587-1590. doi: 10.1016/S0140-6736(16)00650-4

Adibi, J. J., Zhao, Y., Cartus, A. R., Gupta, P., and Davidson, L. A. (2016b). Placental mechanics in the Zika-microcephaly relationship. Cell Host Microbe 20, 9-11. doi: 10.1016/j.chom.2016.06.013

Bayer, A., Lennemann, N. J., Ouyang, Y., Bramley, J. C., Morosky, S., Marques, E. T. Jr., et al. (2016). Type III interferons produced by human placental trophoblasts confer protection against Zika virus infection. Cell Host Microbe 19, 705-712. doi: 10.1016/j.chom.2016.03.008

Blázquez, A. B., Escribano-Romero, E., Merino-Ramos, T., Saiz, J. C., and Martín-Acebes, M. A. (2014). Stress responses in flavivirus-infected cells: activation of unfolded protein response and autophagy. Front. Microbiol. 5:266. doi: 10.3389/fmicb.2014.00266

Burton, G. J., Hempstock, J., and Jauniaux, E. (2001). Nutrition of the human fetus during the first trimester - a review. Placenta 22, S70-S77. doi: 10.1053/plac.2001.0639

Calvet, G., Aguiar, R. S., Melo, A. S., Sampaio, S. A., de Filippis, I., Fabri, A., et al. (2016). Detection and sequencing of Zika virus from amniotic fluid of fetuses with microcephaly in Brazil: a case study. Lancet Infect. Dis. 16, 653-660. doi: 10.1016/S1473-3099(16)00095-5

Carneiro, L. A., and Travassos, L. H. (2016). Autophagy and viral diseases transmitted by Aedes aegypti and Aedes albopictus. Microbes Infect. 18, 169-171. doi: 10.1016/j.micinf.2015.12.006

Chahar, H. S., Bao, X., and Casola, A. (2015). Exosomes and their role in the life cycle and pathogenesis of RNA viruses. Viruses 7, 3204-3225. doi: 10.3390/v7062770

Chifenti, B., Locci, M. T., Lazzeri, G., Guagnozzi, M., Dinucci, D., Chiellini, F., et al. (2013). Autophagy-related protein LC3 and Beclin-1 in the first trimester of pregnancy. Clin. Exp. Reprod. Med. 40, 33-37. doi: 10.5653/cerm.2013.40.1.33

Curtis, S., Jones, C. J., Garrod, A., Hulme, C. H., and Heazell, A. E. (2013). Identification of autophagic vacuoles and regulators of autophagy in villous trophoblast from normal term pregnancies and in fetal growth restriction. J. Matern. Fetal Neonatal Med. 26, 339-346. doi: 10.3109/14767058.2012.733764

de Castro e Paula, L. A., and Hansen, P. J. (2008). Ceramide inhibits development and cytokinesis and induces apoptosis in preimplantation bovine embryos. Mol. Reprod. Dev. 75, 1063-1070. doi: 10.1002/mrd.20841

Dong, Y. S., Yang, J. S., Zhang, H., and He, J. G. (2005). N-1530A, a neutral sphinogominase inhibitor from metabolites of microorganisms. Chin. Pharm. J. 40, 1514-1516.

Fader, C. M., Sánchez, D., Furlán, M., and Colombo, M. I. (2008). Induction of autophagy promotes fusion of multivesicular bodies with autophagic vacuoles in k562 cells. Traffic 9, 230-250. doi: 10.1111/j.1600-0854.2007.00677.x

Faria, N. R., Azevedo Rdo, S., Kraemer, M. U., Souza, R., Cunha, M. S., Hill, S. C., et al. (2016). Zika virus in the Americas: early epidemiological and genetic findings. Science 352, 345-349. doi: 10.1126/science.aaf5036

Farias, K. J., Machado, P. R., Muniz, J. A., Imbeloni, A. A., and da Fonseca, B. A. (2015). Antiviral activity of chloroquine against dengue virus type 2 replication in Aotus monkeys. Viral Immunol. 28, 161-169. doi: 10.1089/vim.2014.0090

Fedson, D. S. (2013). Treating influenza with statins and other immunomodulatory agents. Antiviral Res. 99, 417-435. doi: 10.1016/j.antiviral.2013.06.018

Fuchs, R., and Ellinger, I. (2004). Endocytic and transcytotic processes in villous syncytiotrophoblast: role in nutrient transport to the human fetus. Traffic 5, 725-738. doi: 10.1111/j.1600-0854.2004.00221.x writing. SY conducted the literature search and drafted the manuscript.

\section{ACKNOWLEDGMENTS}

This work was funded by the Preeminent Youth Fund of Sichuan Province (2015JQO045).

Gong, J. S., and Kim, G. J. (2014). The role of autophagy in the placenta as a regulator of cell death. Clin. Exp. Reprod. Med. 41, 97-107. doi: $10.5653 /$ cerm.2014.41.3.97

Hamel, R., Dejarnac, O., Wichit, S., Ekchariyawat, P., Neyret, A., Luplertlop, N., et al. (2015). Biology of Zika virus infection in human skin cells. J. Virol. 89, 8880-8896. doi: 10.1128/JVI.00354-15

Li, C., Xu, D., Ye, Q., Hong, S., Jiang, Y., Liu, X., et al. (2016). Zika virus disrupts neural progenitor development and leads to microcephaly in mice. Cell Stem Cell 19, 120-126. doi: 10.1016/j.stem.2016.04.017

Li, J., Liu, K., Liu, Y., Xu, Y., Zhang, F., Yang, H., et al. (2013). Exosomes mediate the cell-to-cell transmission of IFN- $\alpha$-induced antiviral activity. Nat. Immunol. 14, 793-803. doi: 10.1038/ni.2647

Martín-Acebes, M. A., Blázquez, A. B., and Saiz, J. C. (2015). Reconciling West Nile virus with the autophagic pathway. Autophagy 11, 861-864. doi: $10.1080 / 15548627.2015 .1037062$

Miner, J. J., Cao, B., Govero, J., Smith, A. M., Fernandez, E., Cabrera, O. H., et al. (2016). Zika virus infection during pregnancy in mice causes placental damage and fetal demise. Cell 165, 1081-1091. doi: 10.1016/j.cell.2016.05.008

Mlakar, J., Korva, M., Tul, N., Popović, M., Poljšak-Prijatelj, M., Mraz, J., et al. (2016). Zika virus associated with microcephaly. N. Engl. J. Med. 374, 951-958. doi: 10.1056/NEJMoa1600651

Mor, G. (2016). Placental inflammatory response to Zika virus may affect fetal brain development. Am. J. Reprod. Immunol. 75, 421-422. doi: 10.1111/aji.12505

Noronha, L. d., Zanluca, C., Azevedo, M. L., Luz, K. G., and Santos, C. N. (2016). Zika virus damages the human placental barrier and presents marked fetal neurotropism. Mem. Inst. Oswaldo Cruz 111, 287-293. doi: 10.1590/0074-02760160085

Nour, A. M., Li, Y., Wolenski, J., and Modis, Y. (2013). Viral membrane fusion and nucleocapsid delivery into the cytoplasm are distinct events in some flaviviruses. PLoS Pathog. 9:e1003585. doi: 10.1371/journal.ppat.1003585

Parihar, S. P., Guler, R., Khutlang, R., Lang, D. M., Hurdayal, R., Mhlanga, M. M., et al. (2014). Statin therapy reduces the Mycobacterium tuberculosis burden in human macrophages and in mice by enhancing autophagy and phagosome maturation. J. Infect. Dis. 209, 754-763. doi: 10.1093/infdis/jit550

Pirooz, S., He, S., O’Connell, D., Khalilzadeh, P., Yang, Y., and Liang, C. (2014). Viruses customize autophagy protein for efficient viral entry. Autophagy 10 1355-1356. doi: 10.4161/auto.29075

Ponpuak, M., Mandell, M. A., Kimura, T., Chauhan, S., Cleyrat, C., and Deretic, V. (2015). Secretory autophagy. Curr. Opin. Cell Biol. 35, 106-116. doi: 10.1016/j.ceb.2015.04.016

Quicke, K. M., Bowen, J. R., Johnson, E. L., McDonald, C. E., Ma, H., O’Neal, J. T., et al. (2016). Zika virus infects human placental macrophages. Cell Host Microbe 20, 83-90. doi: 10.1016/j.chom.2016.05.015

Rolfe, A. J., Bosco, D. B., Wang, J., Nowakowski, R. S., Fan, J., and Ren, Y. (2016). Bioinformatic analysis reveals the expression of unique transcriptomic signatures in Zika virus infected human neural stem cells. Cell Biosci. 6, 42. doi: 10.1186/s13578-016-0110-x

Saji, F., Samejima, Y., Kamiura, S., and Koyama, M. (1999). Dynamics of immunoglobulins at the feto-maternal interface. Rev. Reprod. 4, 81-89. doi: 10.1530 /ror.0.0040081

Schiebler, M., Brown, K., Hegyi, K., Newton, S. M., Renna, M., Hepburn, L., et al. (2015). Functional drug screening reveals anticonvulsants as enhancers of mTOR,-independent autophagic killing of Mycobacterium tuberculosis through inositol depletion. EMBO Mol. Med. 7, 127-139. doi: $10.15252 / \mathrm{emmm} .201404137$ 
Sharma, M., Bhattacharyya, S., Nain, M., Kaur, M., Sood, V., Gupta, V., et al. (2014). Japanese encephalitis virus replication is negatively regulated by autophagy and occurs on LC3-I- and EDEM1-containing membranes. Autophagy 10, 1637-1651. doi: 10.4161/auto.29455

Shoji-Kawata, S., Sumpter, R., Leveno, M., Campbell, G. R., Zou, Z., Kinch, L., et al. (2013). Identification of a candidate therapeutic autophagy-inducing peptide. Nature 494, 201-206. doi: 10.1038/nature11866

Toth, F. D., Mosborg-Petersen, P., Kiss, J., Aboagye-Mathiesen, G., Zdravkovic, M., Hager, H., et al. (1994). Antibody-dependent enhancement of HIV-1 infection in human term syncytiotrophoblast cells cultured in vitro. Clin. Exp. Immunol. 96, 389-394. doi: 10.1111/j.1365-2249.1994.tb 06040.x

Tricou, V., Minh, N. N., Van, T. P., Lee, S. J., Farrar, J., Wills, B., et al. (2010). A randomized controlled trial of chloroquine for the treatment of dengue in Vietnamese adults. PLoS Negl. Trop. Dis. 4:e785. doi: 10.1371/journal.pntd. 0000785

Vakifahmetoglu-Norberg, H., Xia, H. G., and Yuan, J. (2015). Pharmacologic agents targeting autophagy. J. Clin. Invest. 125, 5-13. doi: 10.1172/JCI 73937

Vandergaast, R., and Fredericksen, B. L. (2012). West Nile Virus (WNV) replication is independent of autophagy in mammalian cells. PLoS ONE 7:e45800. doi: 10.1371/journal.pone.0045800
Yazdani Brojeni, P., Matok, I., Garcia Bournissen, F., and Koren, G. (2012). A systematic review of the fetal safety of interferon alpha. Reprod. Toxicol. 33, 265-268. doi: 10.1016/j.reprotox.2011.11.003

Zumla, A., Rao, M., Wallis, R. S., Kaufmann, S. H., Rustomjee, R., Mwaba, P., et al. (2016). Host-directed therapies for infectious diseases: current status, recent progress, and future prospects. Lancet Infect Dis. 16, e47-63. doi: 10.1016/S1473-3099(16)00078-5

Conflict of Interest Statement: The authors declare that the research was conducted in the absence of any commercial or financial relationships that could be construed as a potential conflict of interest.

The reviewer IEC and handling Editor declared their shared affiliation and the handling Editor states that the process nevertheless met the standards of a fair and objective review.

Copyright (c) 2017 Zhang, Li and Yuan. This is an open-access article distributed under the terms of the Creative Commons Attribution License (CC BY). The use, distribution or reproduction in other forums is permitted, provided the original author(s) or licensor are credited and that the original publication in this journal is cited, in accordance with accepted academic practice. No use, distribution or reproduction is permitted which does not comply with these terms. 